Original article

\title{
Effect of paclitaxel treatment on cellular mechanics and morphology of human oesophageal squamous cell carcinoma of South African origin in 2D and 3D environments
}

Martin Kiwanuka ${ }^{1}$, Ghodeejah Higgins ${ }^{1}$, Silindile Ngcobo $^{2}$, Juliet Nagawa ${ }^{1}$, Dirk M. Lang ${ }^{3}$, Muhammad H. Zaman ${ }^{4,5}$, Neil H. Davies ${ }^{2}$, Thomas Franz ${ }^{1,6^{*}}$

${ }^{1}$ Division of Biomedical Engineering, Department of Human Biology, University of Cape Town, South Africa

${ }^{2}$ Cardiovascular Research Unit, Christiaan Barnard Division of Cardiothoracic Surgery, University of Cape Town, South Africa

${ }^{3}$ Division of Cell Biology, Department of Human Biology, University of Cape Town, South Africa

${ }^{4}$ Department of Biomedical Engineering, Boston University, MA, United States of America

${ }^{5}$ Howard Hughes Medical Institute, Chevy Chase, MD, United States of America

${ }^{6}$ Bioengineering Science Research Group, Engineering Sciences, University of Southampton, United Kingdom

* Corresponding author

Department of Human Biology

Faculty of Health Sciences

University of Cape Town

Private Bag X3

Observatory 7935

Email: thomas.franz@uct.ac.za

\section{ORCID}

Martin Kiwanuka: 0000-0002-9971-6740

Ghodeejah Higgins: 0000-0003-3576-9031

Juliet Nagawa: 0000-0002-3712-9647

Silindile Ngcobo:

Dirk M. Lang:

Muhammad H. Zaman: 0000-0002-3723-5431

Neil H. Davies: 0000-0003-0432-4515

Thomas Franz: 0000-0002-1504-3842 


\begin{abstract}
During chemotherapy, structural and mechanical changes in malignant cells have been observed in several cancers, including leukaemia, pancreatic and prostate cancer. Such cellular changes may act as physical biomarkers for chemoresistance and cancer recurrence. This study aimed to determine how exposure to paclitaxel affects the intracellular stiffness of human oesophageal cancer of South African origin in vitro. A human oesophageal squamous cell carcinoma cell line WHCO1 was cultured on glass substrates (2D) and in collagen gels (3D) and exposed to paclitaxel for up to 48 hours. Cellular morphology and stiffness were assessed with confocal microscopy, visually aided morpho-phenotyping image recognition, and mitochondrial particle tracking microrheology at 24 and 48 hours. In the 2D environment, the intracellular stiffness was higher for the paclitaxel-treated than for untreated cells at 24 and 48 hours. In the 3D environment, the paclitaxel-treated cells were stiffer than the untreated cells at 24 hours, but no statistically significant differences in stiffness were observed at 48 hours. In 2D, paclitaxel-treated cells were significantly larger at 24 and 48 hours and more circular at 24 but not at 48 hours than the untreated controls. In 3D, there were no significant morphological differences between treated and untreated cells. The shape heterogeneity was lower for paclitaxel-treated than untreated cells in 3D and similar for the treated and untreated groups in 2D. Future studies with patient-derived primary cancer cells and prolonged drug exposure will help identify physical cellular biomarkers to detect chemoresistance onset and assess therapy effectiveness in oesophageal cancer patients.
\end{abstract}

\title{
Keywords
}

Oesophageal cancer; chemotherapy; chemoresistance; physical biomarker; mechanobiology; collagen; mitochondrial particle tracking microrheology; image recognition 


\section{Insight}

Mechanical changes in cancer cells brought on by chemotherapeutic drug exposure offer possible physical biomarkers to detect chemoresistance and cancer recurrence. This first study on human oesophageal squamous cell carcinoma of South African origin indicated that in vitro paclitaxel treatment induced stiffening and enlarging of the malignant cells in twodimensional environments at 24 and 48 hours. In physiologically more relevant threedimensional collagen matrices, however, the paclitaxel treatment led to cellular stiffening at 24 hours but softening after that, without significant changes in cellular size at any time. The in vitro environment's influence on experimental outcomes needs to be considered in further studies with prolonged drug exposure and patient-derived primary cancer cells.

\section{Introduction}

In 2018, oesophageal cancer (OC) ranked as the seventh most pervasive and the sixth deadliest cancer with about 579,000 (3.2\% of 18.1 million) cancer incidences and 508,800 (5.3\% of 9.6 million) deaths reported globally [1]. OC is categorized into oesophageal squamous cell carcinoma (OSCC) and oesophageal adenocarcinoma (OAC), the two most prevalent histological subgroups. OSCC originates from the flat cells lining the upper twothirds of the oesophagus, whereas OAC occurs in the lower third of the oesophagus and generally develops from Barrett mucosa [2]. OAC is most prevalent in high-income countries. In contrast, OSCC is the most prevalent subtype in South-East and Central Asia and Southern and Eastern Africa and is responsible for approximately $90 \%$ of all the oesophageal cancer cases [2].

Oesophageal cancer is typically asymptomatic initially, and most patients present at a late stage when their chances of survival are dismal. As a result, OC has poor treatment outcomes, 
with a five-year survival rate reported at about $15 \%$ to $25 \%$ [3]. OC treatment options include endoscopic or surgical therapy and perioperative chemoradiotherapy [4]. Chemotherapy or chemoradiotherapy are indicated for patients with locally advanced OC or metastases in the lymph nodes. The chemotherapeutic drugs for OC treatment include carboplatin [5], paclitaxel [5, 6], cisplatin [6, 7], fluorouracil [7], and oxaliplatin [8]. The clinical effectiveness of chemoradiation therapy are associated with treatment failure, mainly due to radiation and chemoresistance $[9,10]$, the leading causes of OC recurrence $[11,12]$. Therefore, there is a need to monitor chemoradiotherapy effectiveness and detect radiation resistance, chemoresistance and OC recurrence.

Exposure to chemotherapeutic drugs has been reported to increase cell stiffness in several cancers such as leukaemia [13], pancreatic cancer [14] and prostate cancer [15]. Cellular stiffening has been ascribed to cytoskeletal changes during exposure to chemotherapeutic drugs that aim to induce cell death, for example, by inhibiting cytoskeletal reorganization required for cell division and proliferation [16-18].

Structural and mechanical changes in cancer cells associated with chemotherapy offer possible physical biomarkers to detect chemoresistance and cancer recurrence. However, there are no studies on the effect of chemotherapeutic treatment on cell mechanics of oesophageal cancer.

Hence, this study aimed to determine how exposure to the chemotherapeutic drug paclitaxel in vitro changes the intracellular stiffness and morphology of human oesophageal squamous cell carcinoma of South African origin in two-dimensional (2D) and three-dimensional (3D) environments. 


\section{Materials and methods}

\subsection{Cell culture}

A human oesophageal squamous cell carcinoma (OSCC) cell line, WHCO1, derived from biopsies of South African patients [19], was used in this study. Cells were maintained in 25 $\mathrm{cm}^{2}$ tissue culture flasks (Coster, Corning Life Science, Acton, MA) in a humidified incubator at $37^{\circ} \mathrm{C}$ and $5 \% \mathrm{CO}_{2}$. The cells were cultured in DMEM (Dulbecco's Modified Eagle Medium, SIGMA, Life Science, USA) supplemented with $10 \%$ foetal bovine serum (FBS, Gibco, Life Technologies) and 1\% (v/v) streptomycin-penicillin stock (Gibco, Life Technologies).

\subsection{Chemotherapeutic drug treatment}

For the 2D experiments, cells were grown on $35 \mathrm{~mm}$ glass-bottom dishes (Greiner Bio-One, Germany) for 24 hours and kept in a humidified incubator at $37^{\circ} \mathrm{C}$ and $5 \% \mathrm{CO}_{2}$. The cells were then treated with $2 \mu \mathrm{M}$ of paclitaxel (Teva Pharmaceuticals Pty Ltd) in fresh growth media for up to 48 hours.

For the 3D experiments, cells were grown as single cells in $3 \mathrm{D}$ collagen I gels at a final concentration of $2 \mathrm{mg} / \mathrm{ml}$ and treated with paclitaxel for up to 48 hours. Briefly, $1.4 \times 10^{5}$ cells/10 $\mu 1$ were combined with equal amounts of Type I Rat Tail collagen (BD Bioscience, San Jose, CA) and neutralizing solution (100 mM HEPES dissolved in 2xPBS, pH 7.4, SIGMA Life Science), and further diluted in 1xPBS until the desired $2 \mathrm{mg} / \mathrm{ml}$ collagen concentration was reached. A collagen volume of $50 \mu \mathrm{l}$ was then gelled on $35 \mathrm{~mm}$ glassbottom dishes kept in a humidified incubator at $37^{\circ} \mathrm{C}$ and $5 \% \mathrm{CO}_{2}$ for an hour. After complete collagen gelation, the embedded cells were treated with $2 \mu \mathrm{M}$ of paclitaxel in fresh growth media for up to 48 hours. 
Vehicle control experiments with ethanol alone $(0.014 \%[\mathrm{v} / \mathrm{v}]$, maximum volume used in the drug treatment experiments) were carried out for the $2 \mathrm{D}$ experiments to rule out the effect of ethanol on the cells. The ethanol-treated cells were found to have similar mean squared displacement (MSD) and power-law coefficient $\alpha$ curves compared with the untreated cells. Ethanol treatment was therefore not performed in the 3D experiments.

\subsection{Mitochondrial particle tracking microrheology}

After the 24 and 48 hours of paclitaxel treatment, MitoTracker Green solution (Life Technologies, Carlsbad, CA) was incubated with the cells for 30 minutes (2D) and 45 minutes (3D) at $37^{\circ} \mathrm{C}$ and $5 \% \mathrm{CO}_{2}$ before the microrheology experiments. $100 \mathrm{nM}$ and 400 $\mathrm{nM}$ of MitoTracker green solution in fresh growth media were used to fluorescently label the mitochondria for the cells in 2D and 3D matrices, respectively. The use of endogenous and abundant mitochondria as tracer particles has been validated for short delay times against exogenously injected nano-particles [20].

\subsubsection{Time-lapse image acquisition}

Time-lapse images were acquired for the cells that survived paclitaxel treatment using a confocal microscope (Zeiss LSM 880 Airy-scan, Germany) with a monochrome chargecoupled device (CCD) camera and a 63 X 1.4 NA oil immersion objective. Cells were maintained in the incubation chamber of the confocal microscope at $37^{\circ} \mathrm{C}$ and $5 \% \mathrm{CO}_{2}$ throughout the imaging sessions. ZEN software was used to capture the time-lapse images. During the imaging sessions, single cells were located and tracked for 140 and 150 seconds (approximately seven frames per second) for cells in 2D and 3D, respectively.

For the cells in $3 \mathrm{D}$, only single cells near the centre of the gel were imaged to eliminate the boundary effects. No large focal drifts were observed as individual cells were imaged for only a maximum of 150 seconds. Time-lapse images were captured for at least ten individual 
cells in each condition. Cells that seemed to be dividing were eliminated from the imaging experiments and image analysis.

\subsubsection{Image processing and analysis}

For cells containing at least 75 mitochondria, the particle trajectories describing mitochondrial fluctuations within the cytoplasm were extracted from the time-lapse images for each cell using TrackMate, a Fiji Image J plugin [21, 22]. The particle trajectories were converted to time-dependent mean-squared displacement (MSD) using MATLAB (Math Works, Inc., 2020) and MSD-Analyzer class [22, 23] according to:

$$
\left\langle\Delta r^{2}(\tau)_{x y}\right\rangle=\langle[x(t+\tau)-x(t)]+[y(t+\tau)-y(t)]\rangle^{2}
$$

where $\left\langle\Delta \mathrm{r}^{2}(\tau)_{x y}\right\rangle$ is the ensemble-averaged MSD, $\tau$ is the time interval or delay time between the first and last image frame analyzed, and $\mathrm{x}(\mathrm{t})$ and $\mathrm{y}(\mathrm{t})$ are the spatial coordinates of a particle position at time $t$.

For viscoelastic materials, the MSD scales nonlinearly with the delay time $\tau$ according to a power-law relationship, i.e. $\left\langle\Delta \mathrm{r}^{2}(\tau)\right\rangle \sim \tau^{\alpha}[24]$. The power-law coefficient $\alpha=\partial \ln \left\langle\Delta \mathrm{r}^{2}(\tau)\right\rangle / \partial \ln (\tau)$ represents the slope of the logarithmic MSD- $\tau$ curve.

A low MSD value represents constrained particle fluctuations and stiffer, solid material, whereas a high MSD indicates greater particle motility and a more fluid-like material.

The mitochondrial MSD primarily represents the viscoelastic intracellular properties for short delay times, whereas active intracellular motor-driven processes dominate the MSD for long delay times $[25,26]$. The power-law coefficient $\alpha$ helps classify the motion of the particles: $\alpha$ $\approx 1$ corresponds to diffusive motion such as thermal fluctuations in Newtonian fluids, and $\alpha \approx$ 0 represents constrained, sub-diffusive motion such as thermal fluctuations in an elastic material $[26,27]$. 


\subsection{Morphological analysis}

Morphological analysis was performed using the visually aided morpho-phenotyping image recognition (VAMPIRE) algorithm [28]. Briefly, brightfield images of the treated and untreated cells in 2D and 3D were captured using a confocal microscope and then segmented using the Trainable Weka Segmentation plugin in Fiji Image J [29] to identify cell boundaries. The segmented images were imported to VAMPIRE and used to construct a shape analysis model, which was applied to our dataset to analyze the shapes of treated and untreated cells. In addition to determining the fractional abundance of the treated and untreated cells in each shape mode, it was also used to determine the cells' area, perimeter, circularity, and aspect ratio.

\subsection{Data and statistical analysis}

Statistical analysis was carried out using a two-way ANOVA unless otherwise stated. Residual analysis was used to evaluate the assumptions of a two-way ANOVA. Shapiro-Wilk and Levene's tests were used to determine the normality of data and homogeneity of variance, respectively. On detection of statistical significance, posthoc analysis was carried out using Tukey's test. Statistical significance was assumed at $p<.05$. Data are presented as mean \pm standard deviation unless indicated otherwise. Data are available in the online supplement. All statistical analyses were carried out using SPSS for Windows 26.0. (IBM Corp, Armonk, NY). All experiments were run in duplicate on different days. 


\section{Results}

\subsection{Intracellular stiffness in $2 D$ environments}

Mitochondrial particle tracking microrheology (MPTM) experiments were performed to determine the effect of paclitaxel treatment on the intracellular stiffness of cells in 2D environments. Figure 1 shows representative images of paclitaxel-treated, ethanol-treated, and untreated single cells at 24 and 48 hours. The green-stained mitochondria were used as the tracer particles for the MPTM experiments. The mitochondrial fluctuations within the cytoplasm of the cells were tracked for 26,22 and 31 cells in the paclitaxel-treated, ethanoltreated, and untreated group, respectively.
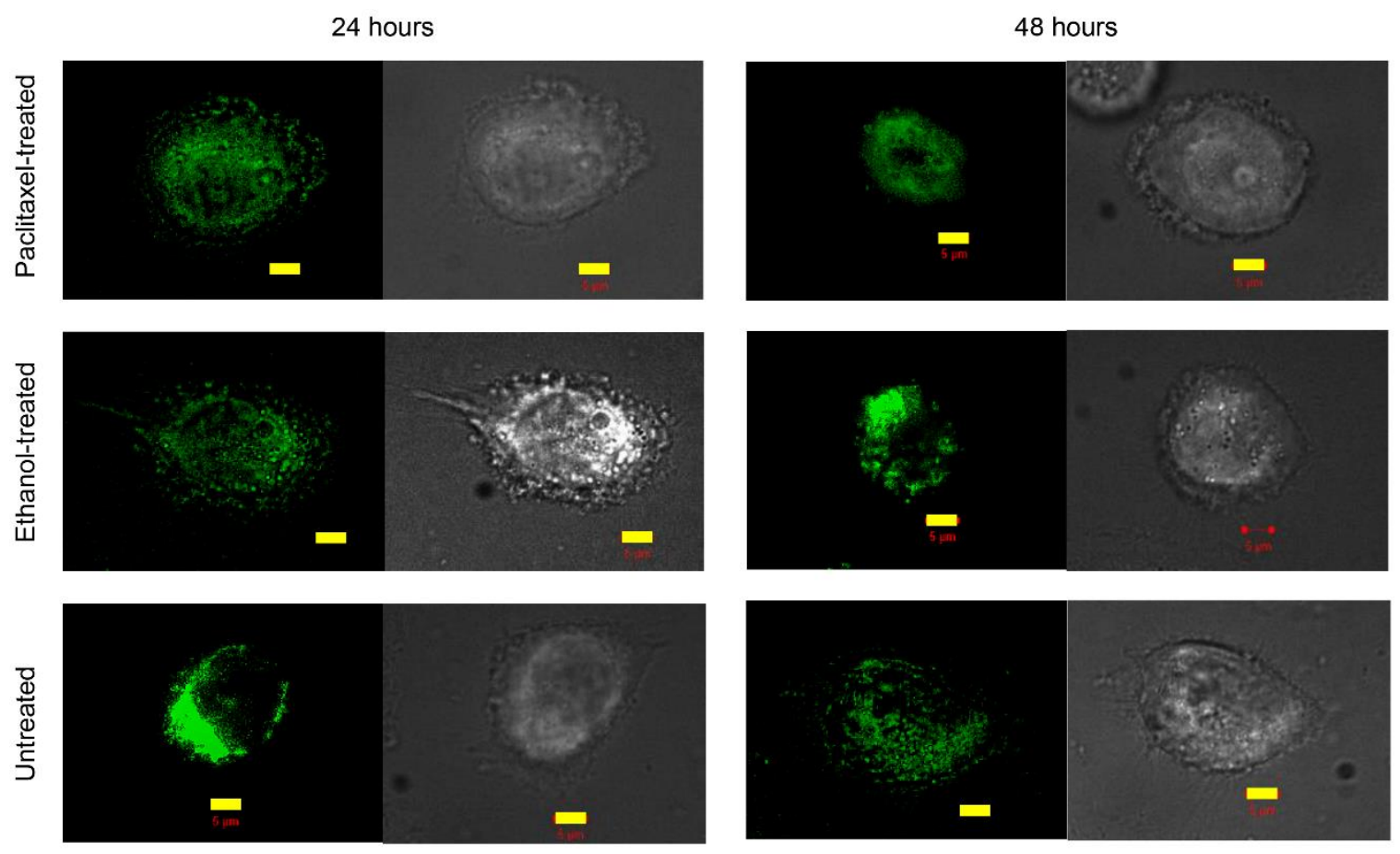

Mitotracker Brightfield

Mitotracker Brightfield

Figure 1: Representative fluorescence and brightfield images of paclitaxel-treated, ethanoltreated and untreated cells in 2D environments after 24 and 48 hours. Scale bars (yellow) represent $5 \mu \mathrm{m}$. 
The paclitaxel-treated cells had significantly higher MSD at 24 hours than at 48 hours at all delay times $(p<0.05)$ except at $\tau=0.14$ seconds (Figure 2 a). Also, the power-law coefficient $\alpha$ of paclitaxel-treated cells was higher at 24 hours than at 48 hours for all delay times, with statistically significant differences only observed between $\tau=0.28$ and 2.1 seconds $(p<0.05)$ (Figure 2 b). There were no statistically significant differences in either MSD or $\alpha$ between 24 and 48 hours for ethanol-treated (Figure $2 \mathrm{c}$ and d) and untreated cells (Figure 2 e and $\mathrm{f}$ ). 

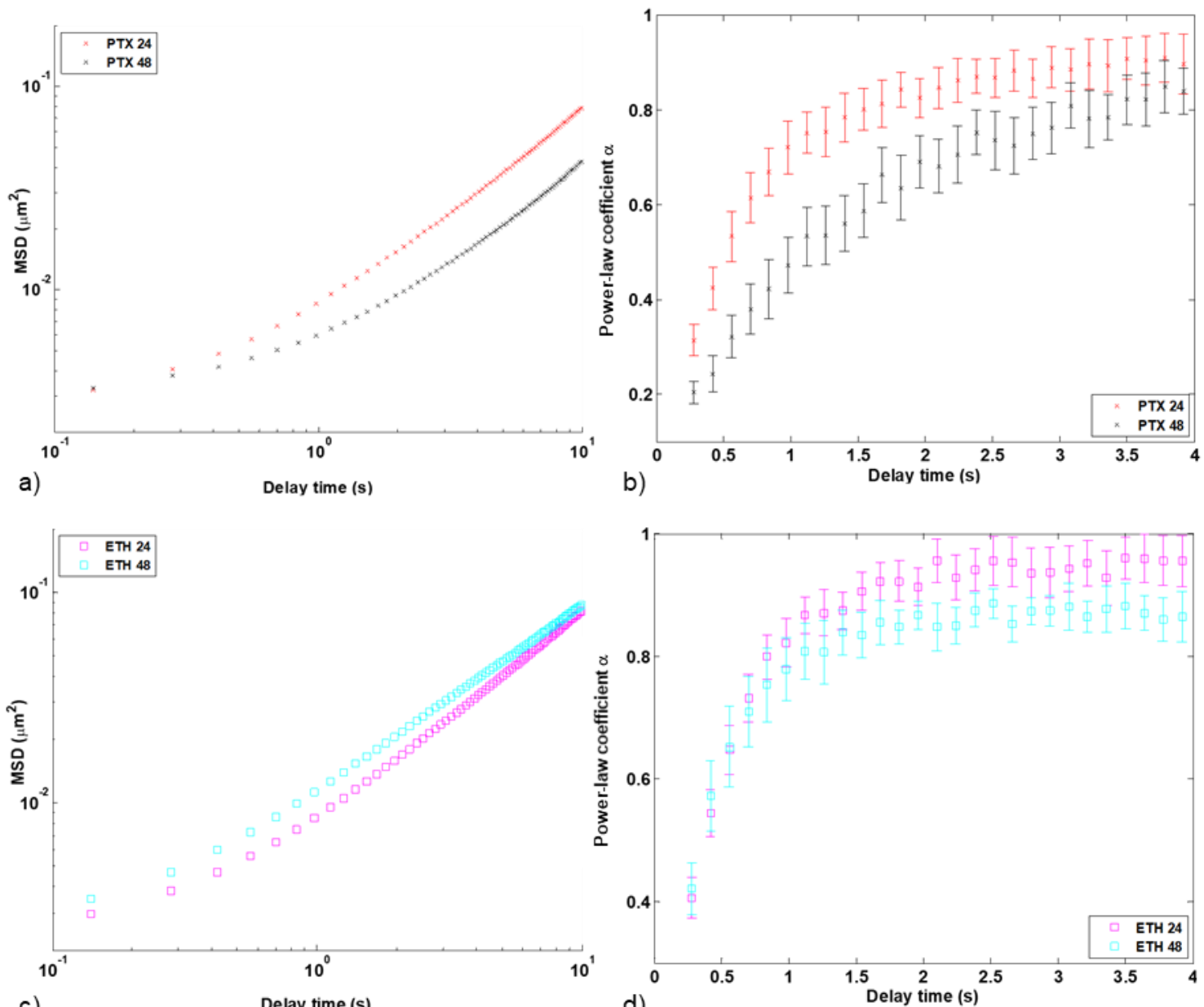

c)

Delay time (s)
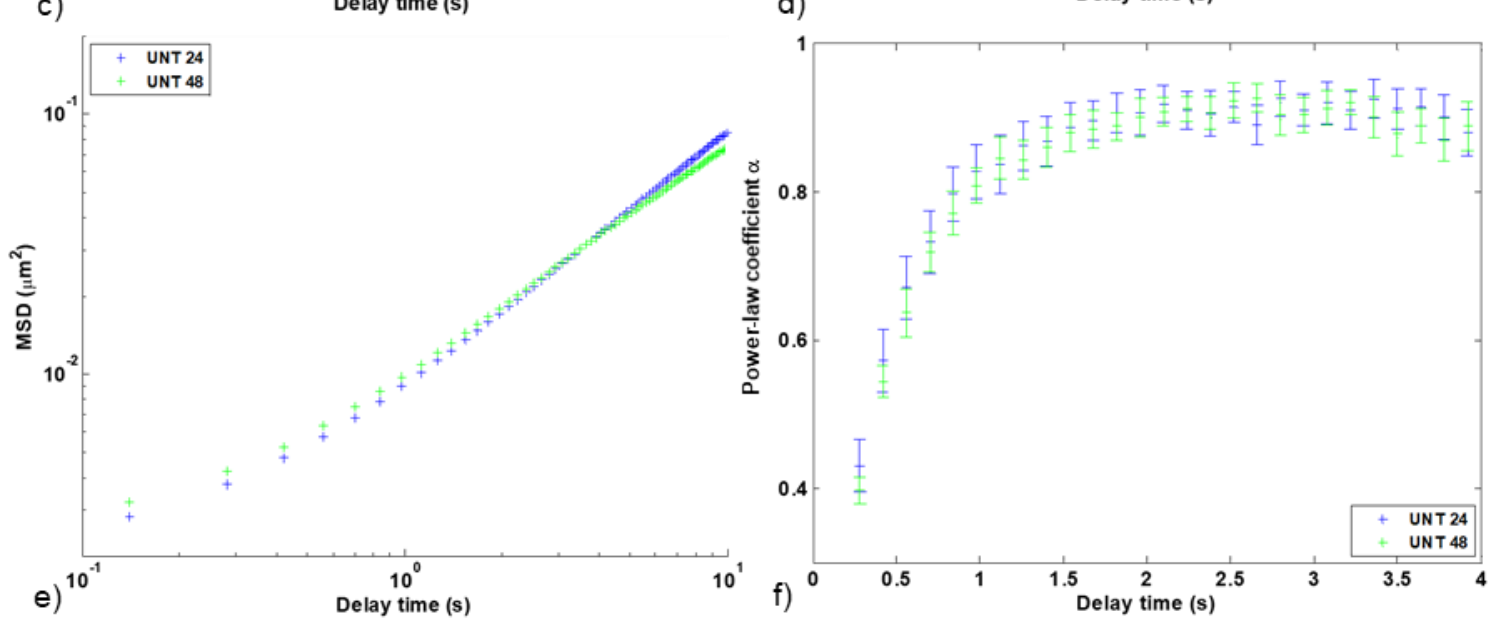

Figure 2: Time-point comparison of microrheological characteristics of cells in 2D environments. MSD versus delay time $\tau$ for paclitaxel-treated (PTX) (a), ethanol-treated (ETH) (c) and untreated cells (UNT) (e) at 24 and 48 hours. Power-law coefficient $\alpha$ versus delay time $\tau$ for paclitaxel-treated (b), ethanol-treated (d) and untreated cells (f) at 24 and 48 hours. Error bars are SEM for one independent repeat experiment ( $\mathrm{n}=2), \mathrm{N}=12$ (PTX 24), 14 (PTX 48), 11 (ETH 24, ETH 48), 17 (UNT 24) and 14 (UNT 48). $N$ refers to the number of cells in each condition. 
At 24 hours of treatment, statistically significant differences in MSD were only observed between paclitaxel-treated and untreated cells at $\tau=0.7$ seconds $(p=0.031)$ (Figure 3 a). The power-law coefficient $\alpha$ of the paclitaxel-treated cells was lower than that of ethanol-treated and untreated cells at all delay times, with statistical significance only observed between paclitaxel-treated and untreated cells at $\tau=0.28$ seconds $(p=0.026)$ (Figure $3 \mathrm{~b})$.

At 48 hours of treatment, the paclitaxel-treated cells had lower MSD than the ethanol-treated $(p<0.05)$ and untreated cells $(p<0.005)$ at all delay times except $\tau=0.14$ seconds. No statistically significant differences in MSD were observed between the ethanol-treated and untreated cells (Figure $3 \mathrm{c}$ ). The power-law coefficient $\alpha$ of the paclitaxel-treated cells was significantly lower than that of the ethanol-treated $(p<0.05)$ and untreated cells $(p<0.005)$, except for delay times between $\tau=3.5$ and 4 seconds. No statistically significant differences in power-law coefficient $\alpha$ were observed between the ethanol-treated and untreated cells (Figure $3 \mathrm{~d}$ ). 


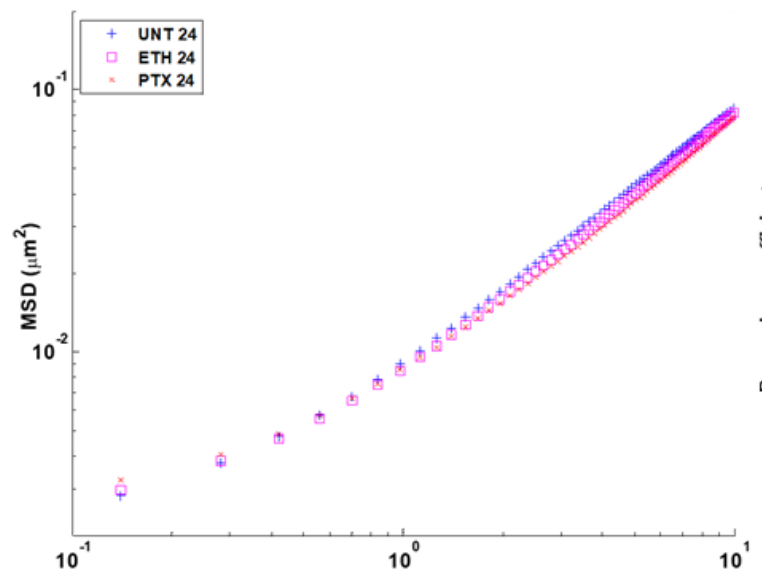

a)

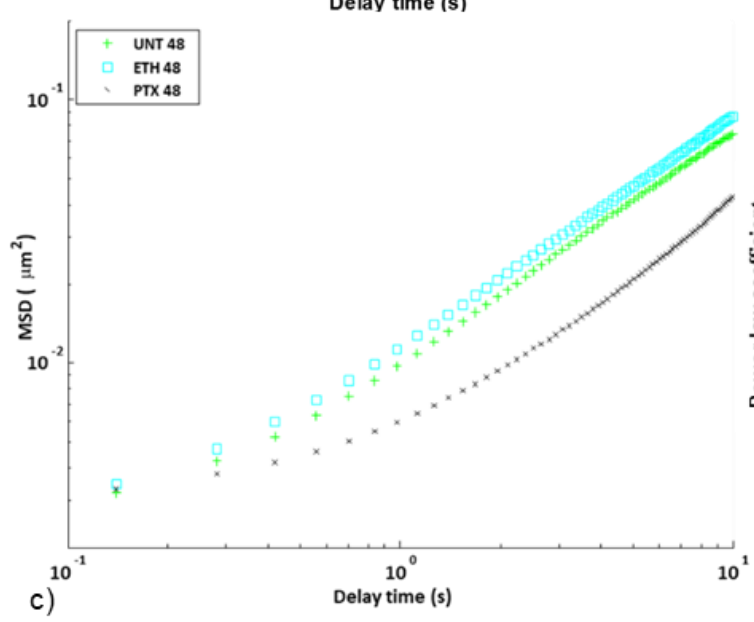

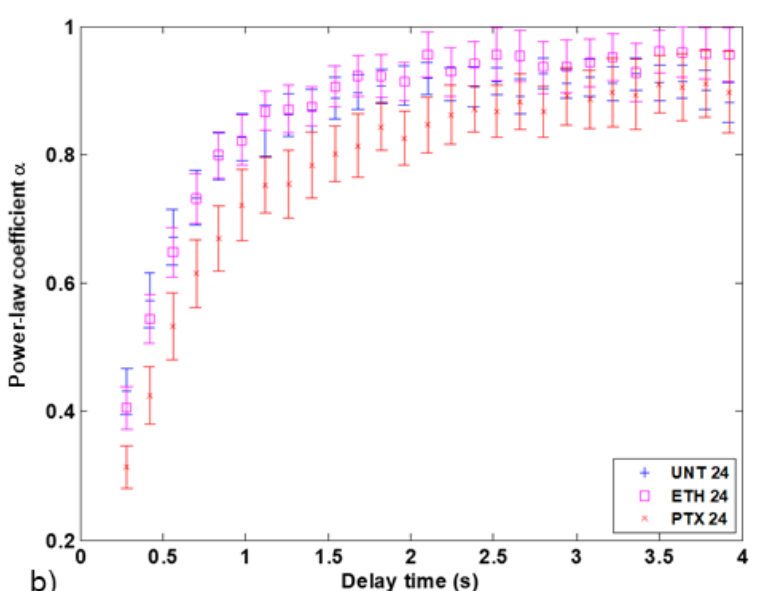

b)

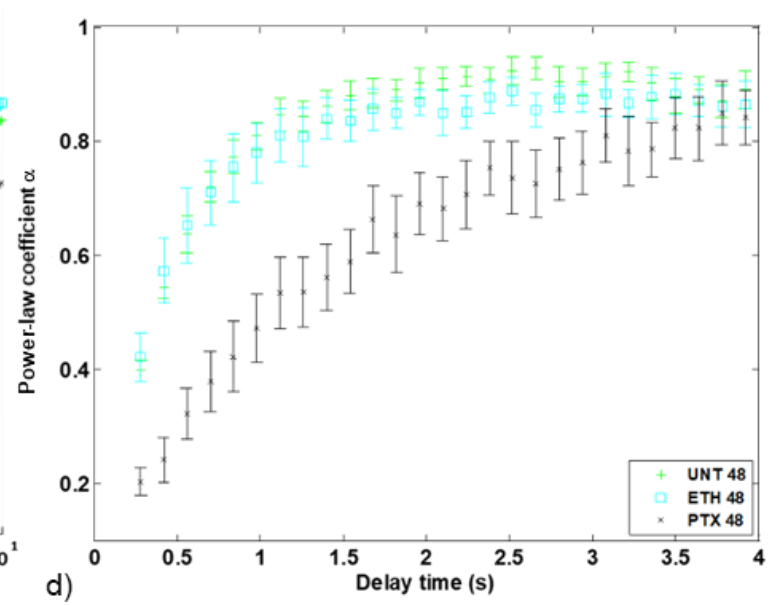

Figure 3: Treatment comparison of microrheological characteristics of cells in 2D environments. MSD versus delay time $\tau$ for paclitaxel-treated (PTX), ethanol-treated (ETH), and untreated cells (UNT) at 24 (a) and 48 hours (c). Power-law coefficient $\alpha$ versus delay time $\tau$ for paclitaxel-treated, ethanol-treated and untreated cells at 24 (b) and 48 hours (d). Error bars are SEM for one independent repeat experiment $(\mathrm{n}=2), \mathrm{N}=17$ (UNT 24), 11 (ETH 24), 12 (PTX 24), 14 (UNT 48), 11 (ETH 48) and 14 (PTX 48).

\subsection{Intracellular stiffness in 3D environments}

Mitochondrial particle tracking microrheology experiments were performed to investigate whether paclitaxel treatment affected the intracellular stiffness of cells embedded as single cells in 3D matrices. Figure 4 shows representative images of the paclitaxel-treated cells and the untreated single cells at 24 and 48 hours of treatment with green-stained mitochondria as 
tracer particles for the MPTM experiments. The displacements of the mitochondria within the cytoplasm were tracked for a total of 31 paclitaxel-treated cells and 32 untreated cells.
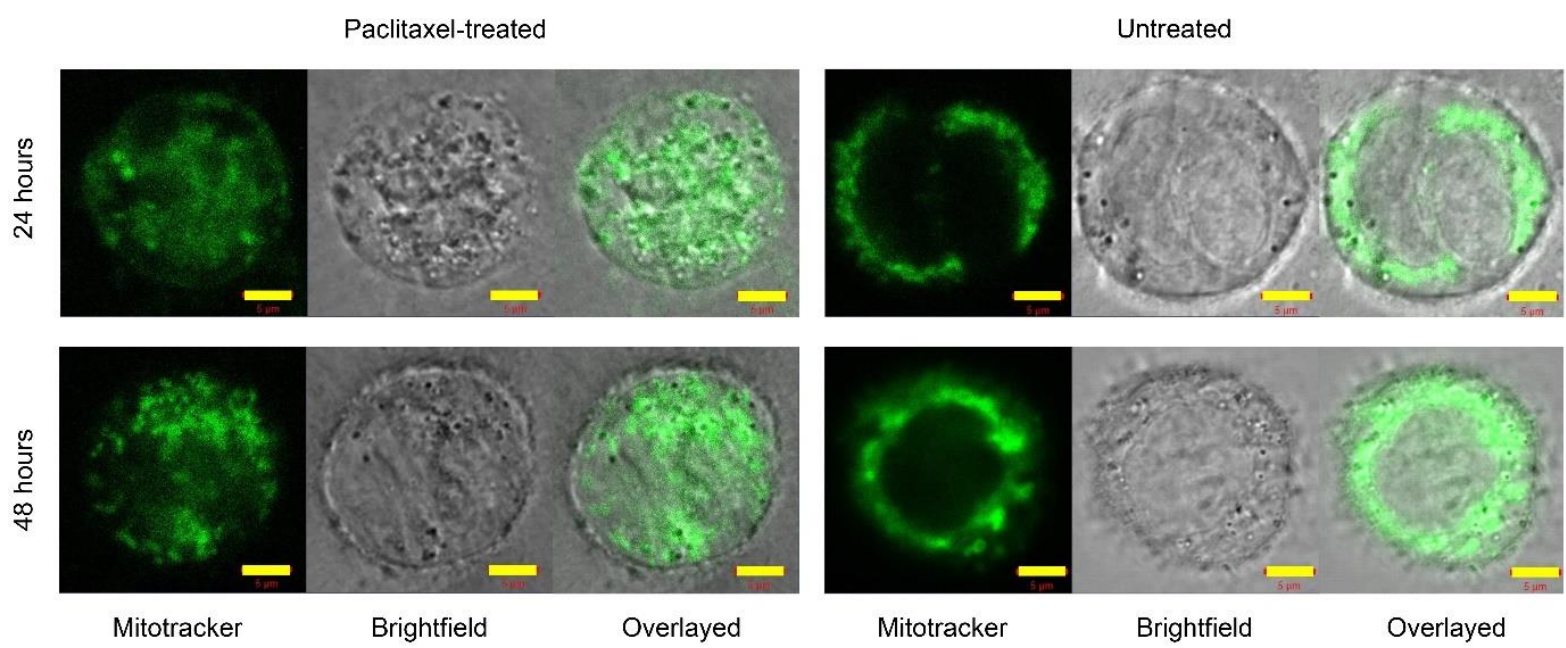

Figure 4: Representative fluorescence, brightfield, and overlay images of paclitaxel-treated, ethanol-treated and untreated single cells in 3D environments after 24 and 48 hours. Scale bars (yellow) represent $5 \mu \mathrm{m}$.

The paclitaxel-treated cells exhibited significantly lower MSD and $\alpha$ at 24 hours than at 48 hours for all delay times $(p<0.05)$ except $\tau=0.15$ seconds for MSD $(p=0.056)($ Figure 5 a) and $\tau=1.2,1.5$, and 2.1 seconds for $\alpha$ (Figure $5 \mathrm{~b}$ ). The untreated cells exhibited higher MSD at 24 hours than at 48 hours at all delay times, with statistical significance observed between $\tau=0.9$ and 3.6 seconds $(p<0.05)$ (Figure 5c). Statistically significant differences in powerlaw coefficient $\alpha$ of the untreated cells between 24 and 48 hours were only observed between $\tau=0.15$ and 0.9 seconds $(p<0.05)($ Figure $5 \mathrm{~d})$.

At 24 hours of treatment, the paclitaxel-treated cells had significantly lower MSD than the untreated cells at all delay times $(p<0.05)$ except between $\tau=0.15$ and 0.3 seconds (Figure 6 a), and $\alpha$ was discernibly lower for the paclitaxel-treated cells than the untreated cells at all delay times $(p<0.05)$ (Figure $6 \mathrm{~b})$. 
At 48 hours of treatment, the paclitaxel-treated cells exhibited higher MSD than the untreated cells at all delay times, with statistical significance between $\tau=0.3$ and 4.2 seconds $(p<$ 0.05) (Figure $6 \mathrm{c}$ ). However, no statistically significant differences in power-law coefficient $\alpha$ were observed between the paclitaxel-treated and untreated cells (Figure $6 \mathrm{~d}$ ).

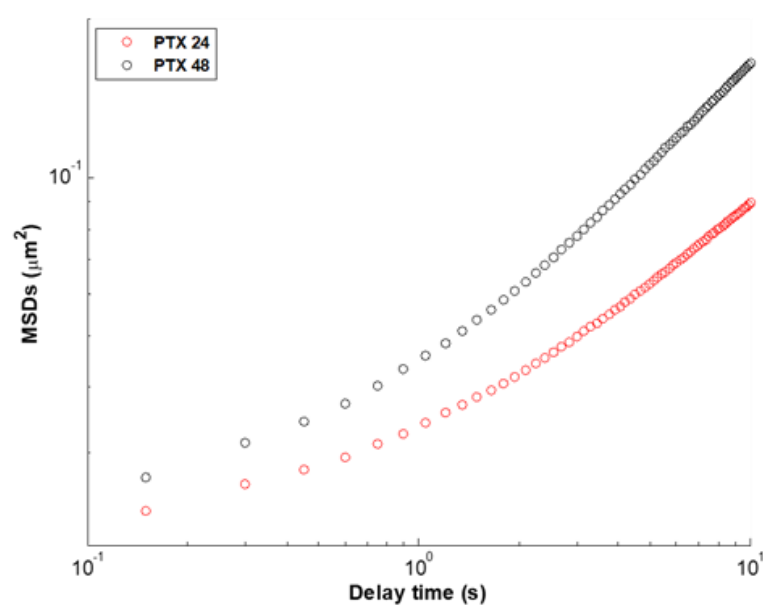

a)

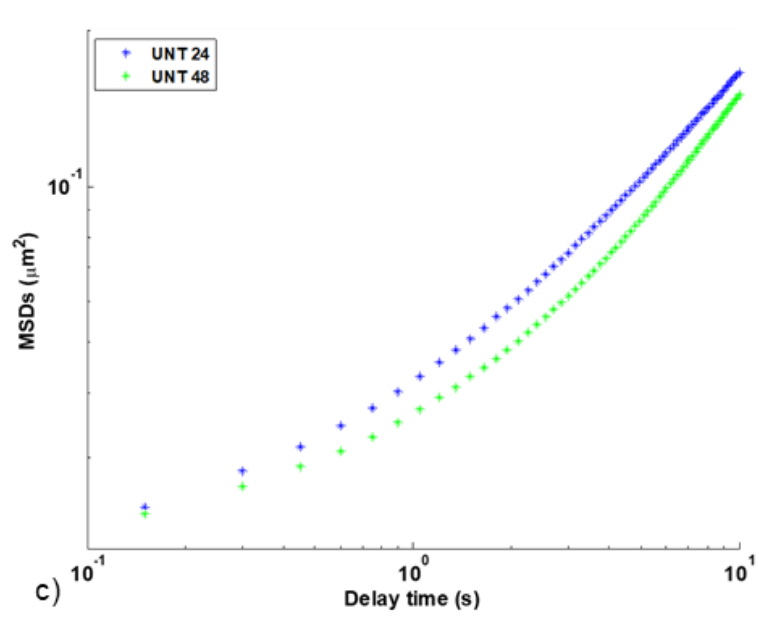

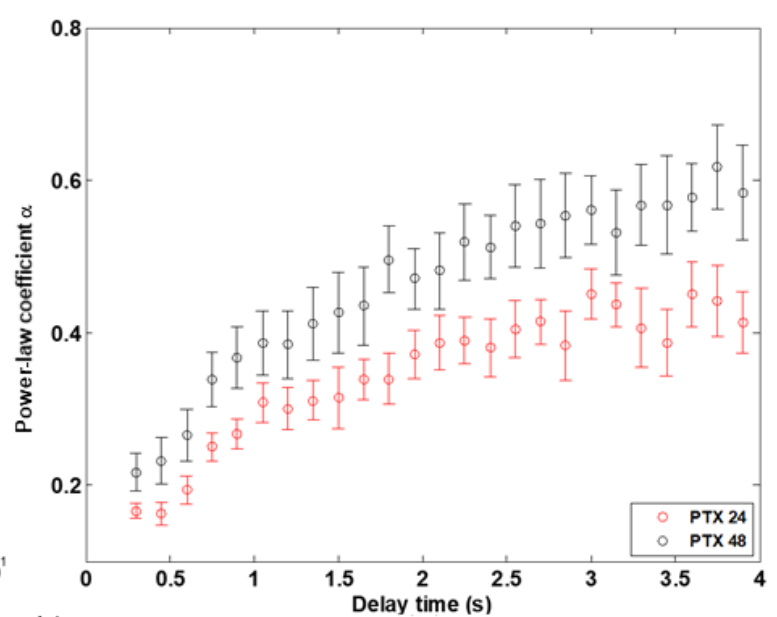

b)

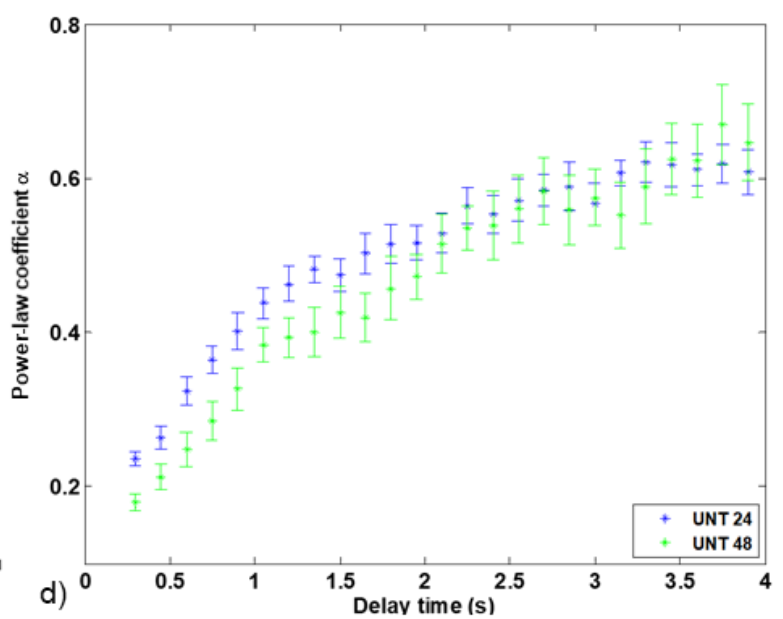

Figure 5: Time-point comparison of microrheological characteristics of cells in 3D environments. MSD versus delay time $\tau$ for (a) paclitaxel-treated cells at 24 (PTX 24) and 48 (PTX 48) hours, and (c) untreated cells at 24 (UNT 24) and 48 (UNT 48) hours. Power-law coefficient $\alpha$ versus delay time $\tau$ for (b) paclitaxel-treated cells at 24 and 48 hours, and (d) untreated cells at 24 and 48 hours. Error bars indicate SEM for one independent repeat experiment ( $\mathrm{n}=2) ; \mathrm{N}=21$ (PTX 24), 10 (PTX 48), 19 (UNT 24) and 13 (UNT 48). 

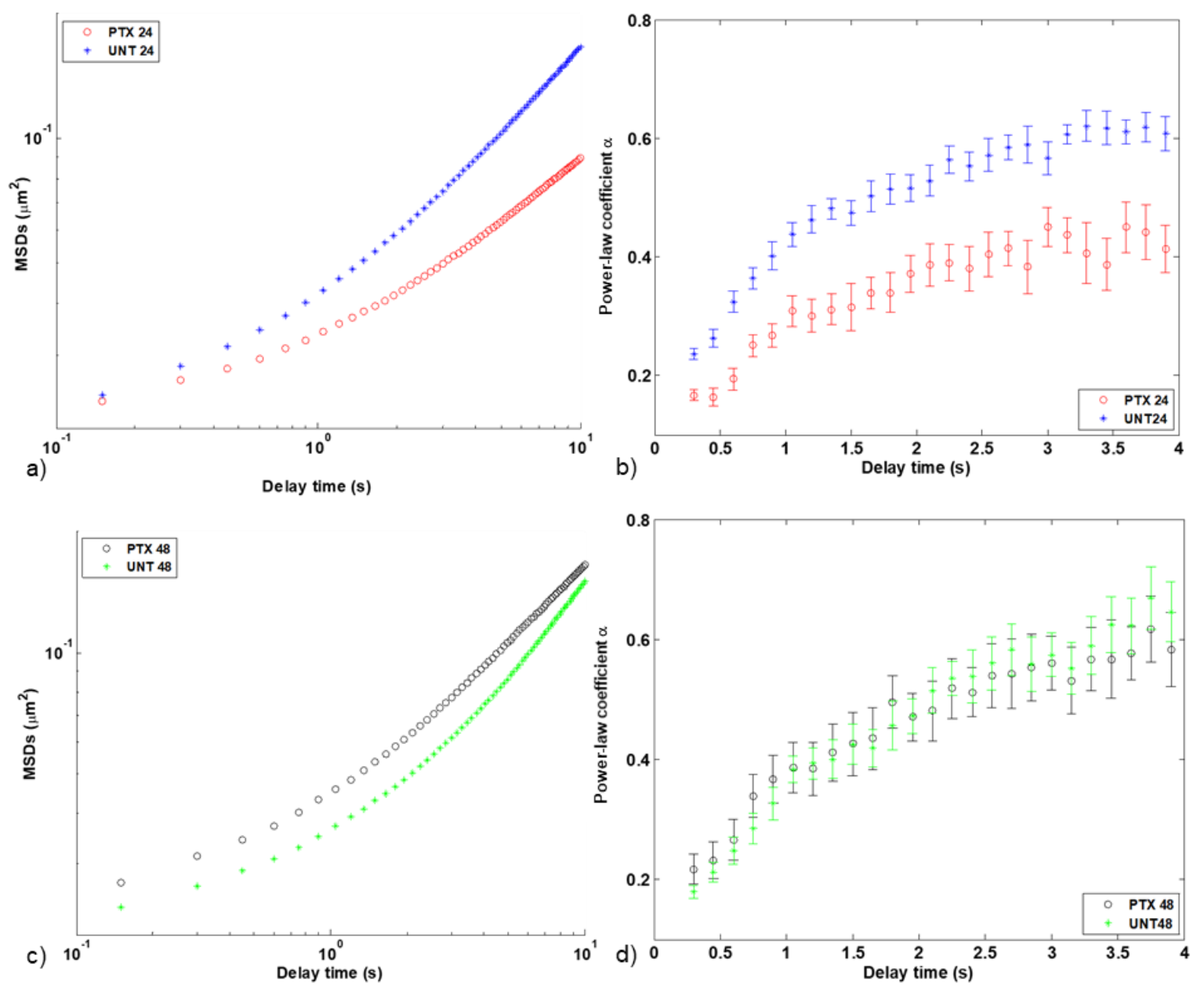

Figure 6: Treatment comparison of microrheological characteristics of cells in 3D environments. MSD versus delay time $\tau$ for paclitaxel-treated (PTX) and untreated cells (UNT) for 24 (a) and 48 hours (c). Power-law coefficient $\alpha$ versus delay time $\tau$ for paclitaxeltreated and untreated cells for 24 (b) and 48 hours (d). Error bars are SEM for one independent repeat experiment $(\mathrm{n}=2), \mathrm{N}=19$ (UNT 24), 21 (PTX 24), 13 (UNT 48) and 10 (PTX 48).

\subsection{Cell morphology}

Using VAMPIRE, cell area, perimeter, circularity, and aspect ratio were determined for 251 treated and untreated cells. Of these, 194 cells were in 2D (49 paclitaxel-treated, and 66 ethanol-treated and 79 untreated cells), and 57 cells were in 3D matrices (26 paclitaxeltreated and 31 untreated cells). A three-way ANOVA was performed to assess the differences 
between groups for the various morphological parameters. Statistical significance was assumed at $p \leq 0.025$.

In 2D environments, the paclitaxel-treated cells had a significantly larger cell area than the untreated $(p<0.005)$ and ethanol-treated cells $(p<0.0005)$ at both 24 and 48 hours (Figure 7 a). The paclitaxel-treated cells had a significantly larger perimeter than the ethanol-treated cells at 24 hours $(p<0.005)$ and the untreated cells at 48 hours $(p<0.005)$. The paclitaxeltreated cells also exhibited a significantly larger perimeter at 48 hours than at 24 hours $(p<$ 0.005) (Figure $7 \mathrm{~b})$. The paclitaxel-treated $(p<0.005)$ and ethanol-treated cells $(p<0.0005)$ were significantly more circular at 24 hours than 48 hours, whereas the opposite was observed for the untreated cells $(p<0.005)$. At 24 hours of treatment, the paclitaxel-treated cells were significantly more circular than the untreated and ethanol-treated cells $(p<$ 0.0005). At 48 hours, no significant differences in circularity were detected between the groups (Figure 7 c). No significant differences in aspect ratio were detected between groups (Figure $7 \mathrm{~d}$ ).

In 3D matrices, no significant differences between groups were observed for cell area, perimeter and aspect ratio (Figure $7 \mathrm{a}, \mathrm{b}$ and d). Circularity was significantly higher in paclitaxel-treated than untreated cells at 24 hours $(p<0.025)$ (Figure $7 \mathrm{c}$ ).

Comparing between 2D and 3D environments, the paclitaxel-treated cells exhibited a significantly larger cell area in 3D than in 2D at both 24 and 48 hours $(p<0.0005)$, and a significantly larger perimeter at 24 hours $(p<0.005)$ but not at 48 hours (Figure 7 a and b). The untreated cells exhibited significantly larger cell area and perimeter in 3D than 2D at 24 and 48 hours $(p<0.0005)$. Cell circularity did not differ significantly between 2D and 3D environments for all treatment conditions and times (Figure 7 c). Significant differences in aspect ratio were only observed between the untreated cells in 2D and 3D at both 24 and 48 
hours $(p<0.025)$ and the paclitaxel-treated cells in 2D and 3D at 48 hours $(p<0.005)$ (Figure $7 \mathrm{~d}$ ).

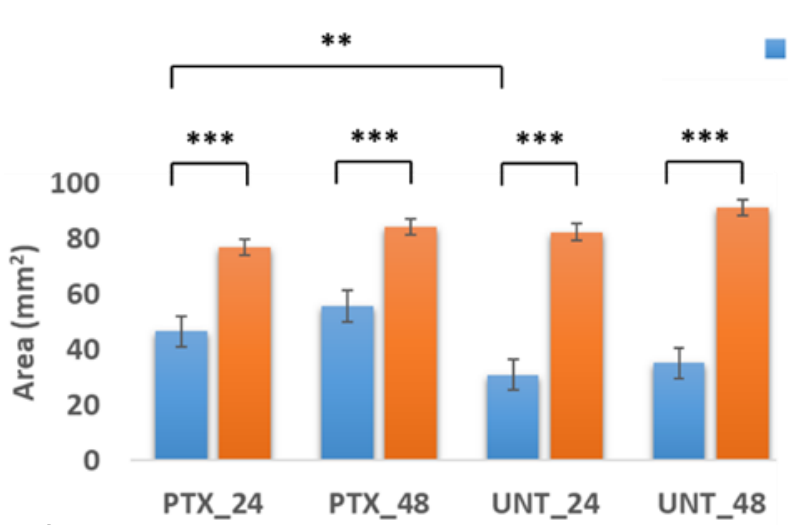

a)

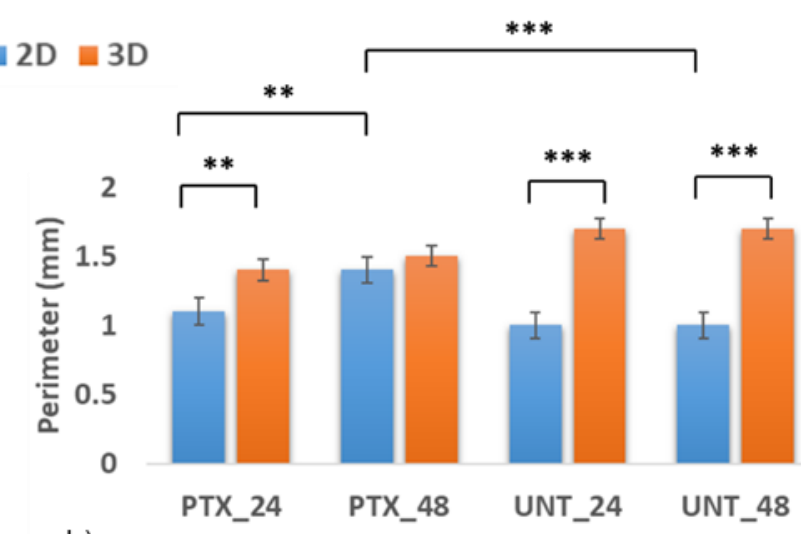

b)

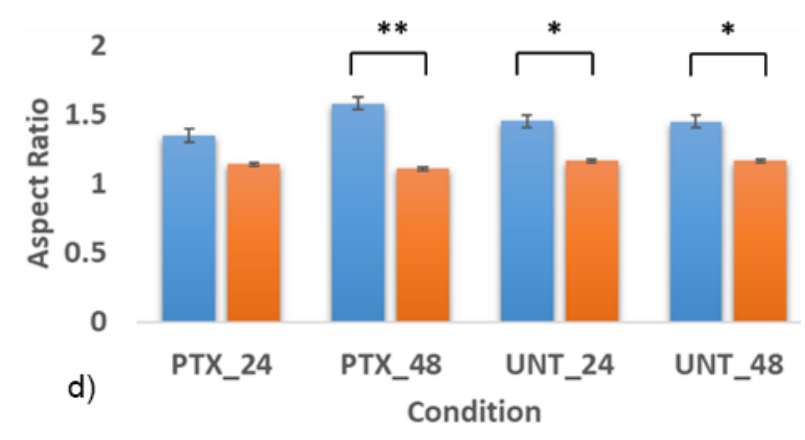

Figure 7: Morphometric data of paclitaxel-treated (PTX) and untreated cells (UNT) in 2D and 3D environments at 24 and 48 hours: Area (a), perimeter (b), circularity (c) and aspect ratio (d). Error bars are SEM for one independent repeat experiment $(n=2), N=27(2 D)+15$ (3D) (PTX_24), $22+11$ (PTX_48), $42+16$ (UNT_24) and $37+15$ (UNT_48).*, ****** indicate $\mathrm{p}<0.025,0.005$, and 0.0005 , respectively.

Ten shape modes were identified from the entire data set of 251 cells using VAMPIRE. These modes represent the inherent shapes of the paclitaxel-treated, ethanol-treated and untreated cells in 2D and 3D matrices (Figure 8). Modes 1 and 2 represent an elongated and circular cell shape, respectively. The modes 3 through 10 represent irregular shapes. The distribution of each shape mode among the paclitaxel-treated, ethanol-treated and untreated 
cells in 2D and 3D matrices were then used to assess the heterogeneity of cell shapes for the different conditions.

In 2D matrices, the paclitaxel-treated cells were morphologically more heterogeneous at 24 hours than at 48 hours, where there were no cells grouped in shape modes 5 and 9 . Most paclitaxel-treated cells at 24 hours were grouped under shape mode $2(18.5 \%)$, whereas those at 48 hours were grouped under shape modes 6, 7, and 10 (18.2\%) (Figure 9 a and b). There were no significant differences in shape heterogeneity of the ethanol-treated cells between 24 and 48 hours. Cell shapes were well distributed across modes, except modes 9 and 6 without cells assigned at 24 and 48 hours. Most of the ethanol-treated cells at 24 hours were grouped under shape modes 4 and 8 (18.6\%), whereas the highest number of ethanol-treated cells at 48 hours were grouped under shape modes 4, 5 and 10 (17.4\%) (Figure $9 \mathrm{c}$ and d).

The untreated cells were also more heterogeneous at 24 hours than at 48 hours, where there were no cells grouped under shape modes 3 and 7. Most of the untreated cells at 24 and 48 hours were grouped under shape modes 7 (19\%) and 1 (23.3\%), respectively (Figure 9 e and f). At both 24 and 48 hours of treatment, no significant differences in heterogeneity were detected between the paclitaxel-treated cells, ethanol, and the untreated cells (Figure 9). In 3D matrices, the paclitaxel-treated cells were significantly more heterogeneous at 24 hours than at 48 hours, where cells were only grouped under four shape modes $(2,4,7$, and 9$)$. Most of the paclitaxel-treated cells at 24 and 48 hours were grouped under shape mode 2 (33.3\% and 36.4\%, respectively) (Figure $10 \mathrm{a}$ and b). The untreated cells were also more heterogeneous at 24 hours than at 48 hours, with most cells grouped under shape mode 9 $(25 \%)$ at 24 hours and mode $2(26.7 \%)$ at 48 hours (Figure $10 \mathrm{c}$ and $\mathrm{d}$ ).

At 24 hours, the paclitaxel-treated cells were less heterogeneous than the untreated cells. The cells were well distributed across all shape modes except modes 8 and 10 for the paclitaxel- 
treated cells and mode 1 for the untreated cells. At 48 hours, the paclitaxel-treated cells were significantly less heterogeneous than the untreated cells. No cells were classified under six shape modes $(1,3,5,6,8$, and 10$)$ for the paclitaxel-treated cells and three shape modes $(1,6$, and 7) for the untreated cells.

(a)

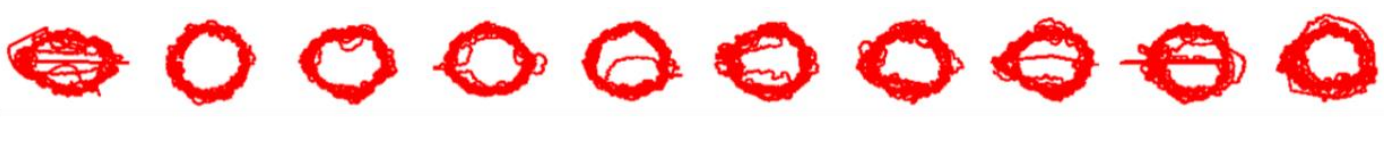

(b)

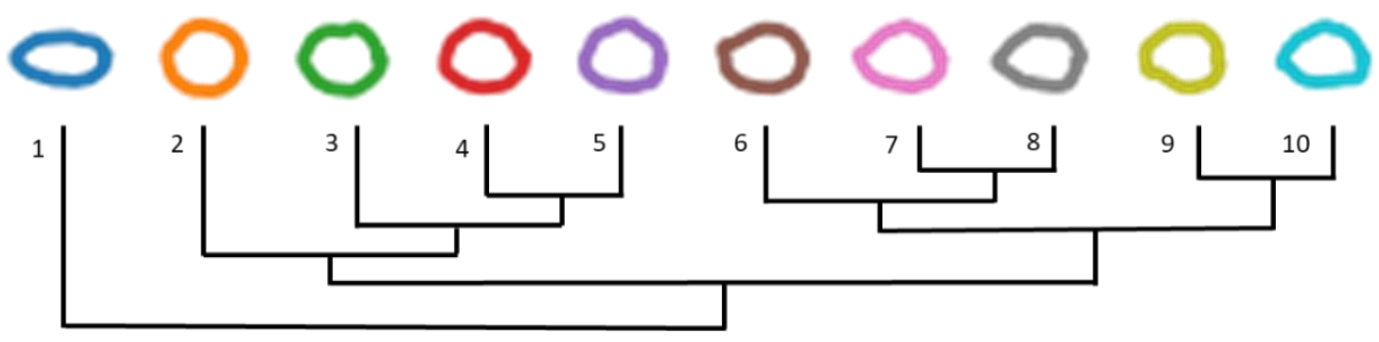

Figure 8: Shape modes obtained for WHCO1 cells in 2D and 3D environments.

(a) Registered objects show actual boundaries of cells selected randomly from individual shape modes. (b) Shape mode dendrogram demonstrates the different shape modes (1 through 10) detected and their similarity (shown by the branches). 

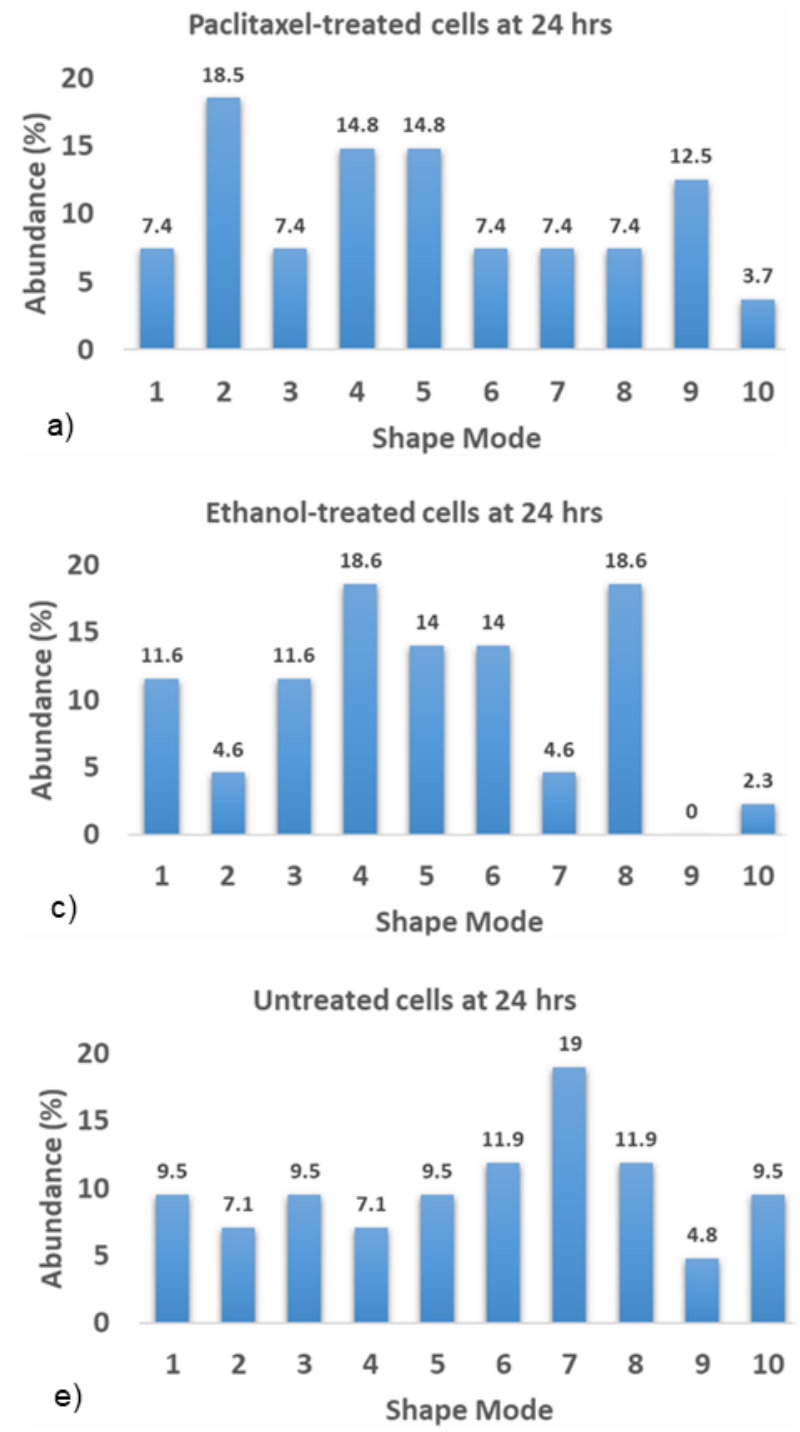
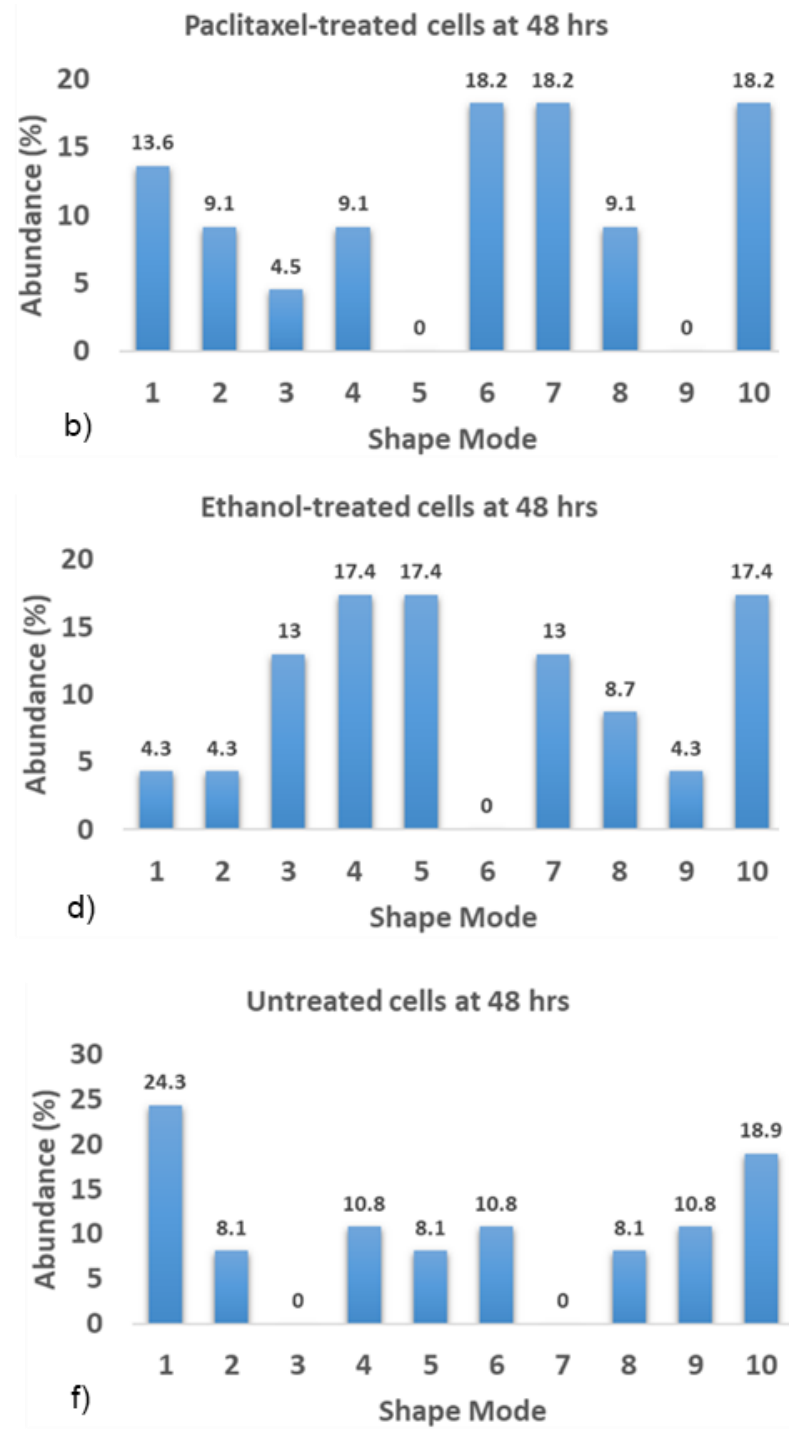

Figure 9: Cellular shape heterogeneity in 2D environments expressed as the relative abundance of cells for shape modes 1 to 10 shown in Figure 8. Paclitaxel-treated cells at 24 (a, $\mathrm{N}=27$ ) and 48 hours (b, N = 22), ethanol-treated cells at 24 (c, $\mathrm{N}=43$ ) and 48 hours (d, $\mathrm{N}=23$ ), untreated cells at $24(\mathrm{e}, \mathrm{N}=42)$ and 48 hours (f, $\mathrm{N}=37)$. See Table S13 in the online supplement for absolute abundance, i.e. the number of cells assigned to each shape mode. 


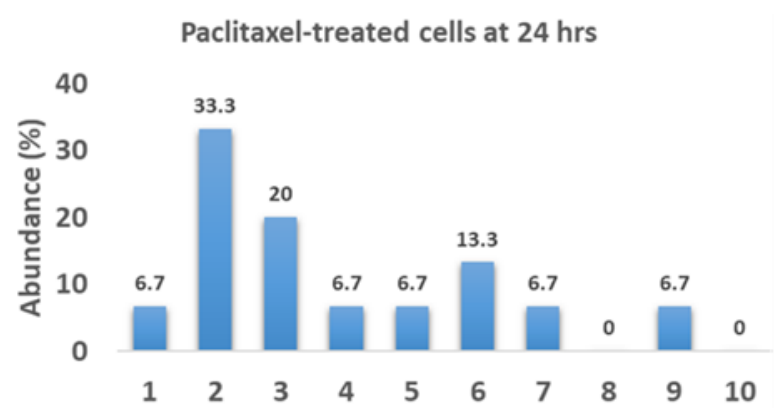

a)

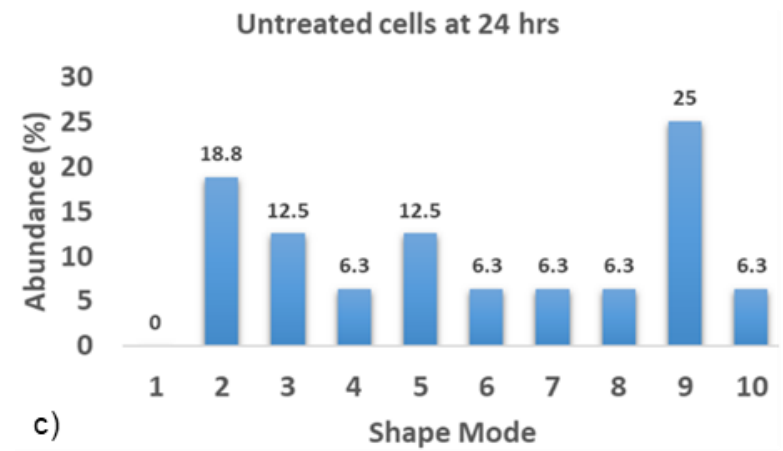

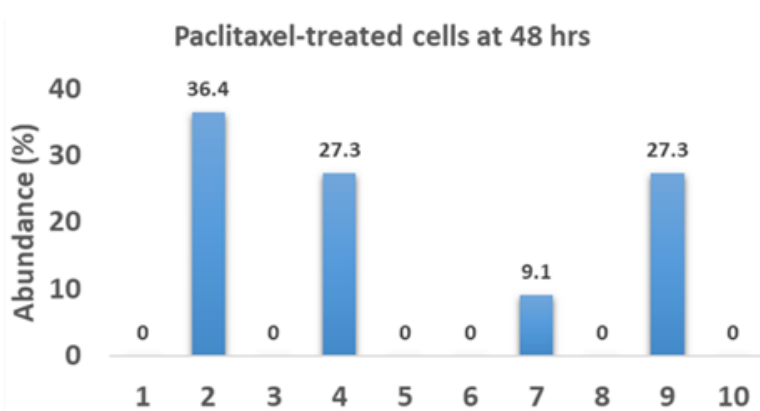

b)

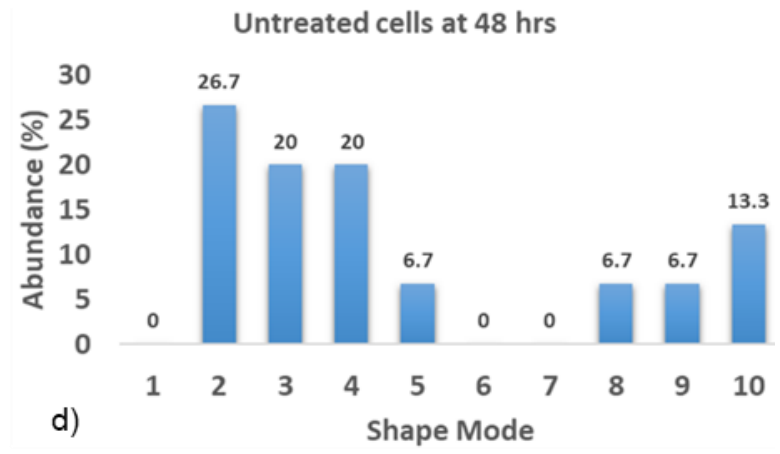

Figure 10: Cellular shape heterogeneity in 3D environments expressed as the relative abundance of cells for shape modes 1 to 10 (Figure 8). Paclitaxel-treated cells at 24 (a, N = $15)$ and 48 hours $(\mathrm{b}, \mathrm{N}=11)$, untreated cells at $24(\mathrm{c}, \mathrm{N}=16)$ and 48 hours $(\mathrm{d}, \mathrm{N}=15)$. Please see Table S13 in the online supplement for absolute abundance, i.e. the number of cells assigned to each shape mode.

\section{Discussion}

\subsection{Intracellular stiffness}

This study characterized the effect of paclitaxel treatment on the intracellular stiffness and morphology of human OSCC cells of South African origin in 2D and 3D environments. The paclitaxel-treated cells in 2D matrices exhibited higher intracellular stiffness (i.e. lower power-law coefficient $\alpha$ ) than the untreated controls at both 24 and 48 hours of treatment. In addition, there was a significant increase in the intracellular stiffness of the paclitaxel-treated cells with an increase in drug exposure time from 24 to 48 hours. The absence of a significant 
stiffness increases with time for the untreated cells confirmed that the prolonged drug exposure led to the stiffening of the paclitaxel-treated cells.

When embedded in 3D collagen matrices, the paclitaxel-treated cells were significantly stiffer than the untreated cells at 24 hours but not 48 hours. The intracellular stiffness significantly decreased for paclitaxel-treated cells, whereas it significantly increased for the untreated cells from 24 to 48 hours. Thus, it is unclear whether and how intracellular stiffness changes in the paclitaxel-treated cells between 24 and 48 hours are related to drug exposure, as intracellular stiffening was also observed for the control at 48 hours.

Results on intracellular stiffening during chemotherapeutic drug exposure in 3D agree with earlier studies on the exposure of K562 and Jurkat leukaemia cells to daunorubicin [13], treatment of PC-3 pancreatic cancer cells with disulfiram, paclitaxel, tomatine, and valproic acid [14] and the effects of cisplatin and docetaxel on prostate cancer cells (PNTIA and 22Rv1) [15].

The stiffening of cancer cells during exposure to chemotherapeutic drugs is attributed to alterations in the cytoskeletal structure since the treatment targets the cytoskeleton to induce cancer cell death. Paclitaxel used in the current study promotes the formation and stabilization of microtubules, reducing the depolymerization and dynamic reorganization of the cytoskeleton required for cell division and proliferation [16-18]. As a result, the cytoskeletal density increases. The stabilized microtubules push the actin cortex outward, leading to cortical strain-hardening [30]. The increase in cytoskeletal density and the strain hardening of the cortex could explain the increase in intracellular stiffness of the paclitaxeltreated cells in the current study. Future studies will confirm the increased cytoskeletal density with drug exposure by measuring total cytoskeletal content through western blotting and immunofluorescence experiments. 


\subsection{Cell morphology}

The morphometric results show that the paclitaxel-treated cells were significantly larger in cell area and perimeter than the untreated controls after 24 and 48 hours in 2D environments. The increased exposure time to paclitaxel of 48 versus 24 hours led to a significant increase in the perimeter (but not the cell area) of paclitaxel-treated cells, whereas the untreated cells did not change the size between 24 and 48 hours. A size increase due to paclitaxel exposure was not observed for cells in 3D environments.

The morphological results for cells in 2D agree with several previous studies demonstrating that cancer cells increase in size due to chemotherapeutic treatment [31-33]. When exposed to paclitaxel, the enlarging of cells can be attributed to the increase in cytoskeletal density based on paclitaxel-promoted formation and stabilization of microtubules, leading to a reduction in their depolymerization and dynamics [16-18]. Another mechanism of cell size increase may be the formation of multiple nuclei within a single cell. Multi-nucleation was observed in the current study (see supplemental Figure S1) and ascribed to the inhibition of cell division by paclitaxel $[32,34]$.

The shape heterogeneity of cells in 3D environments was lower for paclitaxel-treated than untreated cells (10\% at 24 hours and $30 \%$ at 48 hours) and decreased between 24 and 48 hours by $40 \%$ for paclitaxel-treated cells and $20 \%$ for untreated cells. A decrease in shape heterogeneity of $20 \%$ from 24 to 48 hours for paclitaxel-treated and untreated groups, but no difference between the groups, was observed for cells in 2D environments. Hence, paclitaxel exposure affects cell shape in $3 \mathrm{D}$ but not $2 \mathrm{D}$ environments. 


\section{Conclusion}

This study has shown that paclitaxel treatment affects the intracellular stiffness of OSCC cells in both 2D and 3D environments and their morphology (cell area and perimeter when in 2D and heterogeneity when in 3D matrices). This study used a clonal cell line derived from South African OSCC patients; however, future studies with primary human OSCC cells are needed to ascertain the observed changes in the cellular mechanics. Additionally, the OSCC cells in this study were treated with paclitaxel for up to 48 hours. Prolonged exposure to chemotherapeutic drugs in vitro will help characterize the long-term effects of chemotherapeutic drugs on the physical properties of OSCC cell lines and primary cells. These physical characteristics of OSCC cells may complement genetic and molecular markers to assess the effectiveness of chemotherapy and onset of chemoresistance to improve the treatment success in OSCC patients.

\section{Acknowledgements}

We thank the following colleagues from the University of Cape Town: Prof Iqbal Parker from the Department of Integrative Biomedical Sciences for donating WHOC1 cells for this study, Mrs Helen Ilsley from the Cardiovascular Research Unit for technical assistance with cell culturing and histochemistry, and Ms Susan Cooper of the Confocal and Light Microscope Imaging Facility for technical assistance with microscopic imaging. We also thank Prof Denis Wirtz from Johns Hopkins University, Baltimore, Maryland, USA, for providing the visually aided morpho-phenotyping image recognition (VAMPIRE) software. 


\section{Funding}

This work was supported by the Intra-African Academic Mobility Scheme of the European Commission's Education, Audiovisual and Culture Executive Agency (African Biomedical Engineering Mobility Scholarship to MK), the South African Medical Research Council (grant SIR 328148 to TF), and the National Research Foundation of South Africa (grant RA180923361690 to TF). The microscope used in this work was purchased with funds from the Wellcome Trust (grant number 108473/Z/15/Z) and the National Research Foundation of South Africa (grant number UID93197). Any opinion, findings, conclusions, and recommendations expressed in this publication are those of the authors, and therefore, the funders do not accept any liability.

\section{Conflict of Interests}

Conflicts of interest do not exist.

\section{Data availability}

Data supporting the results presented in this article are available on the University of Cape Town's institutional data repository (ZivaHub) under https://doi.org/10.25375/uct.17693510 as Kiwanuka M, Higgins G, Ngcobo S, Nagawa J, Lang DM, Zaman MH, Davies NH, Franz T. Data for "Effect of paclitaxel treatment on cellular mechanics and morphology of oesophageal squamous cell carcinoma of South African origin in 2D and 3D environments". Cape Town, ZivaHub, 2022, DOI: 10.25375/uct.17693510. 


\section{References}

1. Bray F, Ferlay J, Soerjomataram I et al. Global cancer statistics 2018: Globocan estimates of incidence and mortality worldwide for 36 cancers in 185 countries. $C A$ Cancer J Clin, 2018, 68, 394-424.

2. Arnold M, Laversanne M, Brown LM et al. Predicting the future burden of esophageal cancer by histological subtype: International trends in incidence up to 2030. Am J Gastroenterol, 2017, 112, 1247-1255.

3. Pennathur A, Gibson MK, Jobe BA et al. Oesophageal carcinoma. Lancet, 2013, 381, 400-412.

4. Lagergren J, Smyth E, Cunningham D et al. Oesophageal cancer. Lancet, 2017, 390, 2383-2396.

5. van Meerten E, Muller K, Tilanus HW et al. Neoadjuvant concurrent chemoradiation with weekly paclitaxel and carboplatin for patients with oesophageal cancer: A phase ii study. Br J Cancer, 2006, 94, 1389-1394.

6. Polee MB, Eskens FA, van der Burg ME et al. Phase ii study of bi-weekly administration of paclitaxel and cisplatin in patients with advanced oesophageal cancer. Br J Cancer, 2002, 86, 669-673.

7. Gwynne S, Hurt C, Evans M et al. Definitive chemoradiation for oesophageal cancer-a standard of care in patients with non-metastatic oesophageal cancer. Clin Oncol ( $R$ Coll Radiol), 2011, 23, 182-188.

8. van Meerten E, Eskens FA, van Gameren EC et al. First-line treatment with oxaliplatin and capecitabine in patients with advanced or metastatic oesophageal cancer: A phase ii study. Br J Cancer, 2007, 96, 1348-1352.

9. Kim JJ, Tannock IF. Repopulation of cancer cells during therapy: An important cause of treatment failure. Nat Rev Cancer, 2005, 5, 516-525. 
10. Nyhan MJ, O'Donovan TR, Boersma AW et al. Mir-193b promotes autophagy and non-apoptotic cell death in oesophageal cancer cells. BMC Cancer, 2016, 16, 101.

11. Su XD, Zhang DK, Zhang X et al. Prognostic factors in patients with recurrence after complete resection of esophageal squamous cell carcinoma. J Thorac Dis, 2014, 6, 949-957.

12. Zhen Q, Gao LN, Wang RF et al. Lncrna pcat-1 promotes tumour growth and chemoresistance of oesophageal cancer to cisplatin. Cell Biochem Funct, 2018, 36, 27-33.

13. Islam M, Mezencev R, McFarland B et al. Microfluidic cell sorting by stiffness to examine heterogenic responses of cancer cells to chemotherapy. Cell Death and Disease, 2018, 9.

14. Ren J, Huang H, Liu Y et al. An atomic force microscope study revealed two mechanisms in the effect of anticancer drugs on rate-dependent young's modulus of human prostate cancer cells. PLoS One, 2015, 10, e0126107.

15. Raudenska M, Kratochvilova M, Vicar T et al. Cisplatin enhances cell stiffness and decreases invasiveness rate in prostate cancer cells by actin accumulation. Scientific Reports, 2019, 9, 1-11.

16. Weaver BA. How taxol/paclitaxel kills cancer cells. Mol Biol Cell, 2014, 25, 2677 2681.

17. Gascoigne KE, Taylor SS. How do anti-mitotic drugs kill cancer cells? J Cell Sci, 2009, 122, 2579-2585.

18. Fan W. Possible mechanisms of paclitaxel-induced apoptosis. Biochem Pharmacol, 1999, 57, 1215-1221.

19. Veale R, Thornley A. Atypical cytokeratins synthesized by human oesophageal carcinoma cells in culture. South African Journal of Science, 1984, 80, 260.

20. Mak M, Kamm RD, Zaman MH. Impact of dimensionality and network disruption on microrheology of cancer cells in 3D environments. PLoS Comput Biol, 2014, 10, e1003959. 
21. Tinevez J-Y, Perry N, Schindelin J et al. Trackmate: An open and extensible platform for single-particle tracking. Methods, 2017, 115, 80-90.

22. Tinevez J-Y, Herbert S, in Bioimage data analysis workflows, Springer, Cham, 2020, pp. 67-96.

23. Tarantino N, Tinevez JY, Crowell EF et al. Tnf and il-1 exhibit distinct ubiquitin requirements for inducing nemo-ikk supramolecular structures. J Cell Biol, 2014, 204, 231-245.

24. Baker EL, Lu J, Yu D et al. Cancer cell stiffness: Integrated roles of threedimensional matrix stiffness and transforming potential. Biophys $J, 2010,99,2048$ 2057.

25. Arnold M, Soerjomataram I, Ferlay J et al. Global incidence of oesophageal cancer by histological subtype in 2012. Gut, 2015, 64, 381-387.

26. Chan D, Zhou Y, Chui CH et al. Expression of insulin-like growth factor binding protein-5 (igfbp5) reverses cisplatin-resistance in esophageal carcinoma. Cells, 2018, 7.

27. Mason TG, Ganesan K, van Zanten JH et al. Particle tracking microrheology of complex fluids. Physical Review Letters, 1997, 79, 3282-3285.

28. Wu PH, Phillip JM, Khatau SB et al. Evolution of cellular morpho-phenotypes in cancer metastasis. Sci Rep, 2015, 5, 18437.

29. Arganda-Carreras I, Kaynig V, Rueden C et al. Trainable weka segmentation: A machine learning tool for microscopy pixel classification. Bioinformatics, 2017, 33, 2424-2426.

30. Kubitschke H, Schnauss J, Nnetu KD et al. Actin and microtubule networks contribute differently to cell response for small and large strains. New J Phys, 2017, 19, 093003 .

31. Rasbridge SA, Gillett CE, Seymour AM et al. The effects of chemotherapy on morphology, cellular proliferation, apoptosis and oncoprotein expression in primary breast carcinoma. Br J Cancer, 1994, 70, 335-341. 
32. Abubaker K, Latifi A, Luwor R et al. Short-term single treatment of chemotherapy results in the enrichment of ovarian cancer stem cell-like cells leading to an increased tumor burden. Mol Cancer, 2013, 12, 24.

33. Bouzekri A, Esch A, Ornatsky O. Multidimensional profiling of drug-treated cells by imaging mass cytometry. FEBS Open Bio, 2019, 9, 1652-1669.

34. Jordan MA, Wendell K, Gardiner S et al. Mitotic block induced in hela cells by low concentrations of paclitaxel (taxol) results in abnormal mitotic exit and apoptotic cell death. Cancer Res, 1996, 56, 816-825. 